\title{
Writhe in the Stretch-Twist-Fold Dynamo
}

\author{
Mahboubeh Asgari-Targhi ${ }^{\dagger}$ and Mitchell A. Berger ${ }^{\ddagger}$
}

\begin{abstract}
This paper looks at the influence of writhe in the stretch-twist-fold dynamo. We consider a thin flux tube distorted by simple stretch, twist, and fold motions and calculate the helicity and energy spectra. The writhe number assists in the calculations, as it tells us how much the internal twist changes as the tube is distorted. In addition it provides a valuable diagnostic for the degree of distortion. Non mirror-symmetric dynamos typically generate magnetic helicity of one sign on large scales and the opposite sign on small scales. The calculations presented here confirm the hypothesis that the large scale helicity corresponds to writhe and the small scale corresponds to twist. In addition, the writhe helicity spectrum exhibits an interesting oscillatory behavior. The technique of calculating Fourier spectra for the writhe helicity may be useful in other areas of research, for example the study of highly coiled molecules.
\end{abstract}

\section{Introduction}

Magnetic fields are ubiquitous in the universe. The magnetic fields in the Sun, galaxies and planets are generated and maintained by dynamo action, the process in which growth of magnetic field is caused by the motion of electrically conducting fluid, where the kinetic energy is converted into magnetic energy (Beck et al. 1996, Krause 1993, Kronberg 1994, Kulsrud 1999, Ossendrijver 2003, Tobias 2002, Widrow 2003, Yoshizawa et al. 2004, Zweibel and Heiles 1997).

The magnetic field in the Sun rises through its convective zone $(\mathbf{C Z})$ in the form of isolated strands known as flux tubes (Parker 1979). Observations suggest that flux tubes emerging through the photosphere possess internal twist (Bao and Zhang 1998, Pevtsov et al. 1995). This indicates that flux tubes carry electrical current (i.e. they have twisted field lines) prior to emergence. For example, (Leka et al. 1996) have shown that the current increases in proportion to the flux during the period of emergence. The twist in emerging flux tubes could originate deep in the convection zone as part of the dynamo process (Berger and Ruzmaikin 2000, Brandenburg et al. 2002), or could be present due to the effects of Coriolis forces on rising flux (Longcope and Klapper 1997, Longcope et al. 1999, Blackman and Brandenburg 2003). Additional twist may be created in pre-existing flux due to surface motions such as differential rotation or shear motions (van Ballegooijen 1999, DeVore 2000).

The inherent twist in the emerging field lines may provide important information on the nature of the solar dynamo (Rust and LaBonte 2005, Rust and Kumar 1996). We cannot see below the photosphere (although helioseismology (Basu 1997, Howe et al. 2000, Schou et al. 1992, Thompson et al. 2003) provides extensive information on rotational velocities in the interior of the sun). Observing the emergence of twist and other helicity-containing structure (in either newly emerging flux or pre-existing flux) may prove to be an invaluable diagnostic for magnetic field dynamics in the solar dynamo (Brown et al. 1999, Démoulin et al. 2002, Pevtsov et al. 1995, Pevtsov and Latushko 2000, Rust and Kumar 1996).

The nonlinear dynamics of a thin magnetic flux tube rising through the convection zone has been investigated by several authors (Spruit 1981, Choudhuri and Gilman 1987, Archontis et al. 2004, Chou and Fisher 1989, D'Silva and Choudhuri 1993, Fan et al. 1993). Numerical simulations of Spruit's equations suggest that the magnetic flux tube rises from the base of the convection zone in about 2-3 months (Longcope and Klapper 1997). These models follow from applying the ideal MHD equations to a slender tube of flux. The tube is assumed slender in a sense that its cross-sectional radius is negligible compared to both the atmospheric scale height and any scale of variation along the tube. In Longcope and Klapper's model, the presence of some twist is essential in order to formulate a self-consistent picture of a flux tube.

$\dagger$ Mathematics, UCL WC1E 6BT U.K. email: m.asgari@ucl.ac.uk

¥ SECAM, U. of Exeter, EX4 4QF U.K. and Mullard Space Science Laboratory, UCL RH5 6NT U.K. email: m.berger@exeter.ac.uk 
Without twist, a real flux tube would lack integrity and would not behave as a single object for very long (Parker 1979). Linear analysis (Tsinganos 1980) and numerical simulations (Schussler 1979, Longcope et al. 1996) confirm that an untwisted magnetic flux tube is quickly fragmented by hydrodynamic forces. When twisting the flux tube about the axis, the field lines provide a tension that helps to prevent this fragmentation (Moreno-Insertis and Emonet 1996).

The behaviour of thin flux tubes in a turbulent fluid provides excellent geometrical insights into the dynamo process. The stretch-twist-fold (STF) dynamo (see Figure 1) is a simple qualitative example of a fast dynamo (Vainshtein and Zeldovich 1972, Zeldovich et al. 1983, Vainshtein et al. 1997, 1996, Childress and Gilbert 1995, Moffatt and Proctor 1985). This dynamo starts by first stretching a closed flux tube in two dimensions to twice its length. If the flow is incompressible then the volume of the tube is preserved. In the next step, the rope is twisted into a figure 8 and then it is folded so that there are two loops with their fields pointing in the same direction and they occupy the same volume as the original flux tube. The two loops merge through small diffusive effects. This last step is important in the sense that the new field can not easily undo itself and therefore the whole process becomes irreversible. The newly merged loops are topologically the same as the original loop, with the field strength scaled up by a factor of 2 .

After repeating the algorithm $n$ times, the field in the flux tube grows by a factor of $2^{n}$. If one cycle takes time $T$ then the growth rate is $T^{-1} \ln 2$. This makes the dynamo a fast dynamo, where the growth rate does not decrease with decreasing resistivity (Moffatt and Proctor 1985).

The twist part of the cycle allows the field in the folded loop to add coherently rather than canceling. To twist the loop the motions need to leave the plane and go into the third dimension. The degree to which the flow twists the tube is important here. For instance, if the field were twisted too far, or not far enough, then the resulting field would not align itself with the original field. The net angle of rotation is governed by the geometry of the flow. Maggioni and Ricca (2006) give analytic expressions for several classes of curves, including many of the curves resulting from the STF dynamo.

A thin flux tube can be specified by the geometry of its central axis, the radius (and net magnetic flux) of the tube surrounding the axis, and a twist function: how much field lines twist about the axis as a function of position along the axis. We ignore the distribution of flux or twist in the radial direction. The axis geometry can be described locally in terms of two intrinsic quantities, the curvature and torsion. In addition, there are three important global quantities, the twist number, the writhe (Berger and Field 1984, Moffatt and Ricca 1992, Berger and Prior 2006, Maggioni and Ricca 2006) (briefly, a measure of coiling), and the helicity, the measure of linking number of the field lines averaged over all pairs of field lines, and weighted by flux (Moffatt 1969). Longcope and Klapper (1997) have developed a detailed theory of the evolution of a thin flux tube in terms of these geometric quantities. In particular, they allow the distribution of twist to vary along the axis of the tube.

For the purposes of this paper, we make a strong simplification: we assume that the twist will at all times be uniformly distributed. Uniform distribution of twist is valid in an equilibrium situation (Longcope and Klapper 1997, Parker 1979). In a dynamic situation, the assumption will be justified if the time-scale for relaxation to a uniform distribution of twist is small compared to the timescales for evolution of the shape of the tube axis. As redistribution of twist operates at near Alfvén speeds this difference in timescales should be valid for tube motions much slower than the Alfvén speed.

The growing complexity of the field in the STF process can be characterized by the evolution of the magnetic helicity (Vainshtein and Zeldovich 1972). This is discussed in (Brandenburg and Subramanian 2005) and (Gilbert 2002): repeated application of the STF cycle leads to a large scale writhe helicity associated with the repeated crossings of the flux tube and oppositely signed twist helicity at a much smaller scale.

Suppose that the twist process proceeds as in figure 1C (rather than the mirror image process). Gilbert (Gilbert 2002) notes that after folding, the twisted rope has a writhe of approximately $\mathcal{W} \approx-1$. This gives the writhe contribution to the helicity of $H_{\mathrm{wr}} \approx-1$. There is a compensating twist of field lines inside the tube, giving a twist of +1 , and the twist contribution to the helicity is $H_{\mathrm{tw}}=+1$. These figures assume that the fold part of the cycle returns the field to a nearly flat state. If the field shape is more three-dimensional, then the writhe and twist must be calculated more carefully, as will be done below in section 3. The total helicity after one STF step is $H=H_{\mathrm{wr}}+H_{\mathrm{tw}}=0$. Gilbert suggests that the two 
forms of helicity are associated with different scale of the field. The positive twist helicity is associated with structure internal to the tube, presumably at small scales, whereas the negative writhe helicity is related to the coiling of the tube at a larger scale.

Central to the discussion of the large scale dynamo is the so-called alpha effect which explains the generation of a mean field if the turbulence lacks mirror symmetry, e.g. if the flow has kinetic helicity. Large scale dynamos produce small scale helical fields as a waste product that quench the large scale dynamo and hence quench the alpha effect (Brandenburg 2001, Field and Blackman 2002). If most of the helicity of the solar magnetic field is produced by the $\alpha$ effect, then one would expect much of the solar magnetic field to be bi-helical (Blackman and Brandenburg 2003, Blackman and Field 2000, Longcope et al. 1996, Yousef and Brandenburg 2003), in that the field that is generated by the $\alpha$ effect has positive and negative magnetic helicity at different scales, but hardly any net magnetic helicity. To the extent that the helical structure lies in individual tubes (rather than linking between tubes) these different scales of helicity could manifest themselves in twist and writhe structure. Evidence for bi-helical fields can be seen in the fact that bipolar regions are tilted according to Joy's law (D'Silva and Choudhuri 1993, Hale et al. 1919), suggesting the presence of positive (Northern) magnetic helicity in addition to the negative magnetic helicity found in Northern active regions.

On the other hand, if most of the sun's helicity is caused by differential rotation (Berger and Ruzmaikin 2000), the helicity in each hemisphere might have a strong dominant sign (negative in the North). The differential rotation causes segregation of magnetic helicity in physical space, i.e. between north and south, while the $\alpha$ effect causes a segregation of helicity in wavenumber space.

An emerging flux tube can carry helicity as internal twist within the tube, or as writhe (Longcope et al. 2007). To assist in interpreting both dynamo theory and observations of emerging helical structure, we investigate how the decomposition of helicity into twist and writhe (defined in Section 2) relates to the helicity and energy spectra. Consider an STF dynamo with right handed twisting motions (corresponding to the effects of Coriolis forces in the Northern hemisphere of the sun). According to Gilbert (Gilbert 2002) and Brandenburg and Subramanian (Brandenburg and Subramanian 2005), the STF process generates positive helicity at the largest scales, and negative helicity at small scales. The large scale helicity is then interpreted as writhe, while the small scale helicity corresponds to twist. We wish to test this interpretation with explicit calculations.

The plan of this paper is as follows. In section 2, we review the magnetic helicity, twist, and writhe of flux tubes. This section also gives a review of the helicity and energy spectra. In section 3, we provide details of our thin flux tube model and present our calculations. Conclusions will be given in section 4 .

\section{Helicity, twist, and writhe}

\subsection{Definitions}

The Gauss linking number $\mathcal{L}$ measures the linking of two curves (Epple 1998). Similarly, magnetic helicity $H$ can be interpreted as the average linking of pairs of magnetic field lines. We consider a closed tube of magnetic field lines with axial flux $\Phi$. The helicity per unit flux $H / \Phi^{2}$ of the magnetic tube can be divided into two pieces (Berger and Field 1984, Moffatt and Ricca 1992) called the writhe helicity $\mathcal{W}$ and the twist helicity $\mathcal{T}$.

Motions which do not break the curves conserve $\mathcal{L}$, just as ideal motions conserve magnetic helicity $H$. The relation $H / \Phi^{2}=\mathcal{T}+\mathcal{W}$ is analogous to the relation $\mathcal{L}=\mathcal{T}+\mathcal{W}$ known to hold for the edges of a ribbon (Călugăreanu 1961, Pohl 1980, White 1969). The twist $\mathcal{T}$ measures how much a secondary curve (for example one edge of a ribbon) twists about the first (the central axis of the ribbon). Let $s$ denote arc length along a curve, $\mathbf{T}(s)$ the tangent vector, and $\hat{\mathbf{V}}(s)$ a unit vector pointing to the secondary curve. Then

$$
\mathcal{T}=\frac{1}{2 \pi} \oint \mathbf{T} \cdot \hat{\mathbf{V}} \times \frac{\mathrm{d} \hat{\mathbf{V}}}{\mathrm{ds}} \mathrm{ds}
$$



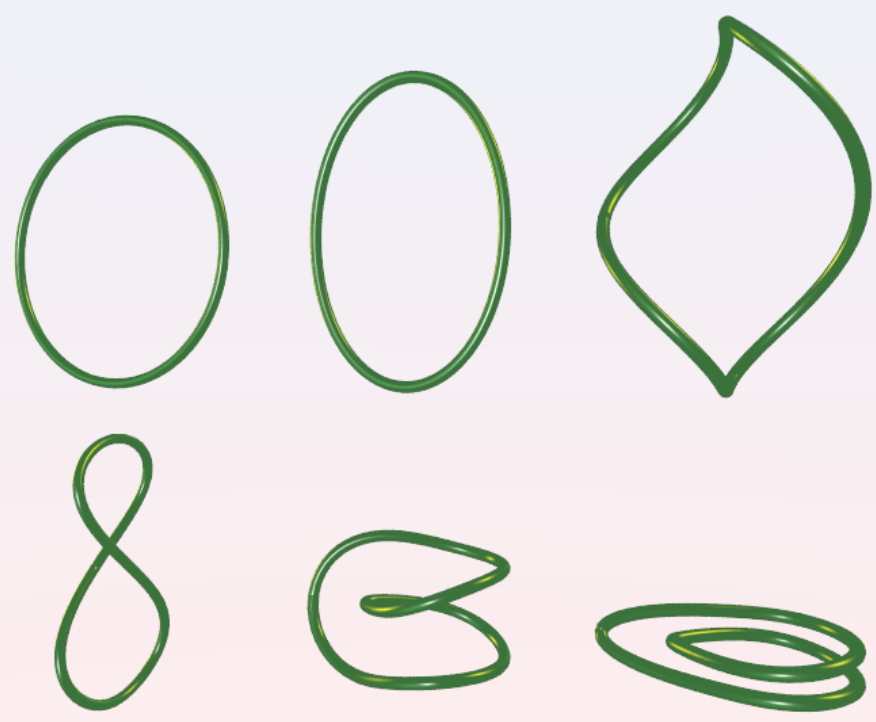

Figure 1.: The stretch-twist-fold process. Top row: an initial circular flux tube $(\mathcal{W}=0)$ is stretched $(\mathcal{W}=0)$ and twisted $(\mathcal{W}=0.295)$. Bottom row: the tube is compressed into a figure $8(\mathcal{W}=0.739)$, folded $(\mathcal{W}=1.12)$, and compressed again $(\mathcal{W}=1.15)$. Writhe can be computed by counting the (signed) number of crossings seen in a plane projection, then averaging over all projection angles. Thus for the figure 8 a positive crossing is seen from $74 \%$ of all projection angles. Note that the last two tubes have a writhe slightly greater than one; from some angles these tubes exhibit two crossings (e.g. if rotated from their present positions by $45^{\circ}$ about the vertical).

For magnetic fields, this twist number measures field aligned current. In particular, if $J_{\|}$is the current parallel to the magnetic field, and $\mu_{0}$ is the vacuum permeability, then

$$
\frac{\mathrm{d} \mathcal{T}}{\mathrm{d} s}=\frac{\mu_{0} J_{\|}}{4 \pi|\mathbf{B}|}
$$

The writhe number $\mathcal{W}$ is defined by the integral

$$
\mathcal{W}=\frac{1}{4 \pi} \oint \oint \frac{\mathbf{x}(s)-\mathbf{x}\left(s^{\prime}\right)}{\left|\mathbf{x}(s)-\mathbf{x}\left(s^{\prime}\right)\right|^{3}} \cdot \frac{\mathrm{d} \mathbf{x}(s)}{\mathrm{d} s} \times \frac{\mathrm{d} \mathbf{x}\left(s^{\prime}\right)}{\mathrm{d} s^{\prime}} \mathrm{d} s \mathrm{~d} s^{\prime}
$$

Simpler and more efficient methods have been found for actually calculating $\mathcal{W}$ (Berger and Prior 2006). Note that $\mathcal{T}$ is defined by a single integral and therefore has a well defined local density. In contrast, $\mathcal{W}$ is defined by a double integral and thus has no local density; it depends on the global geometry of the curve and can not be calculated by adding contributions from individual short sections of the curve.

Because of the conservation of helicity, a reduction in twist will result in an increase in $\mathcal{W}$. Therefore, a change in writhe will have the opposite sign as the change in $\mathcal{T}$. Using the measurement of writhe, we determine the internal twist by

$$
\mathcal{T}=\frac{H}{\Phi^{2}}-\mathcal{W}
$$

The opportunity to change twist into writhe and decrease the energy may result in writhing instabilities where the axis forms helical structures (Longcope and Klapper 1997, Torok and Kleim 2005, 2003, Torok et al. 2004, Valori et al. 2005). 


\section{$2.2 \quad$ Energy and helicity spectra}

The Fourier transforms of the magnetic field and vector potential are (using $\mathbf{B}=\nabla \times \mathbf{A}$ )

$$
\widetilde{\mathbf{B}}_{\mathrm{k}}=\frac{1}{(2 \pi)^{3}} \int \mathbf{B}(\mathbf{x}) \mathrm{e}^{-\mathrm{i} \mathbf{k} \cdot \mathbf{r}} \mathrm{d}^{3} x=-\mathrm{i} \mathbf{k} \times \widetilde{\mathbf{A}}_{\mathrm{k}} .
$$

With this convention, the magnetic energy spectrum is (Moffatt 1978)

$$
M_{\mathrm{k}}=\frac{1}{2} \int_{k-\text { shell }} \widetilde{\mathbf{B}}_{\mathrm{k}}^{*} \cdot \widetilde{\mathbf{B}}_{\mathrm{k}} k^{2} \mathrm{~d} \Omega_{k}
$$

and the helicity spectrum is

$$
H_{\mathrm{k}}=\frac{1}{2} \int_{k-\text { shell }}\left(\widetilde{\mathbf{A}}_{\mathrm{k}}^{*} \cdot \widetilde{\mathbf{B}}_{\mathrm{k}}+\widetilde{\mathbf{A}}_{\mathrm{k}} \cdot \widetilde{\mathbf{B}}_{\mathrm{k}}^{*}\right) k^{2} \mathrm{~d} \Omega_{k}
$$

where $\mathrm{d} \Omega_{k}$ is the solid angle element in Fourier space.

The helicity and energy spectra are normalized so we have

$$
\begin{gathered}
\int_{0}^{\infty} H_{\mathrm{k}} \mathrm{d} k=\langle\mathbf{A} \cdot \mathbf{B}\rangle V \equiv H . \\
\int_{0}^{\infty} M_{\mathrm{k}} \mathrm{d} k=\left\langle\frac{1}{2} \mathbf{B}^{2} \cdot\right\rangle V \equiv M .
\end{gathered}
$$

where $H$ and $M$ are magnetic helicity and magnetic energy, respectively, $V$ is the volume element and the angular brackets denote volume averages.

We can easily show that $H_{\mathrm{k}}$ is gauge invariant: if $\mathbf{A} \rightarrow \mathbf{A}+\nabla \phi$, then $\widetilde{\mathbf{A}}_{\mathrm{k}} \rightarrow \widetilde{\mathbf{A}}_{\mathrm{k}}+\mathrm{i} \tilde{\phi}_{\mathrm{k}} \mathbf{k}$. But as $\nabla \cdot \mathbf{B}=0$, we have $\mathrm{i} \tilde{\phi}_{\mathrm{k}} \mathbf{k} \cdot \widetilde{\mathbf{B}}_{\mathrm{k}}=0$. Thus equation 7 is unchanged by the gauge transformation.

We can decompose the Fourier transformed magnetic vector potential $\widetilde{\mathbf{A}}_{\mathrm{k}}$, into a longitudinal component, $\mathbf{h}^{\|}$, and eigenfunctions $\mathbf{h}^{ \pm}$of the curl operator

$$
\widetilde{\mathbf{A}}_{\mathrm{k}}=a_{\mathrm{k}}^{+} \mathbf{h}_{\mathrm{k}}^{+}+a_{\mathrm{k}}^{-} \mathbf{h}_{\mathrm{k}}{ }^{-}+a_{\mathrm{k}}^{\|} \mathbf{h}_{\mathrm{k}} \|
$$

with

$$
i \mathbf{k} \times \mathbf{h}_{\mathrm{k}}{ }^{ \pm}= \pm k \mathbf{h}_{\mathrm{k}}{ }^{ \pm}, \quad k=|\mathbf{k}|
$$

and

$$
\left\langle\mathbf{h}_{\mathrm{k}}{ }^{* *} \cdot \mathbf{h}_{\mathrm{k}}{ }^{+}\right\rangle=\left\langle\mathbf{h}_{\mathrm{k}}{ }^{*} \cdot \mathbf{h}_{\mathrm{k}}\right\rangle=\left\langle\mathbf{h}_{\mathrm{k}}\left\|^{*} \cdot \mathbf{h}_{\mathrm{k}}\right\|\right\rangle=1,
$$

The longitudinal part $a_{\mathrm{k}}^{\|} \mathbf{h}_{\mathrm{k}} \|$ is parallel to $k$ and vanishes after taking the curl to calculate the magnetic field. (It can also be set to zero by choosing a Coulomb gauge potential, $\nabla \cdot \mathbf{A}=0$.)

The complex coefficients $a_{\mathrm{k}}^{ \pm}(t)$ depend on $k$ and $\mathrm{t}$, and the eigenfunctions $\mathbf{h}_{k}^{ \pm}$, which form an orthonormal set, depend on $k$ (Brandenburg and Subramanian 2005). They are given by

$$
\mathbf{h}_{k}^{ \pm}=\frac{1}{\sqrt{2}} \frac{\mathbf{k} \times(\mathbf{k} \times \mathbf{e}) \mp \mathrm{i} k(k \times \mathbf{e})}{k^{2} \sqrt{1-(\mathbf{k} \cdot \mathbf{e})^{2} / k^{2}}}
$$


where $\mathbf{e}$ is an arbitrary unit vector that is not parallel to $\mathbf{k}$. Using the above relations, we can then write the magnetic helicity spectrum as

$$
H_{\mathrm{k}}=k\left(\left|a_{\mathrm{k}}^{+}\right|^{2}-\left|a_{\mathrm{k}}^{-}\right|^{2}\right) V
$$

and energy spectrum in the form

$$
M_{\mathrm{k}}=\frac{1}{2} k^{2}\left(\left|a_{\mathrm{k}}^{+}\right|^{2}+\left|a_{\mathrm{k}}^{-}\right|^{2}\right) V
$$

where $V$ is the volume of integration. From equations (14) and (15) we have the realizability condition

$$
\frac{1}{2} k\left|H_{\mathrm{k}}\right| \leq M_{\mathrm{k}}
$$

This condition becomes an equality for the eigenfields $\mathbf{h}_{\mathrm{k}}^{+}$and $\mathbf{h}_{\mathrm{k}}^{-}$.

The energy and helicity spectra are closely related to the Fourier transform of the two-point correlation function $R_{i j}(\mathbf{r})$ of the magnetic field (Moffatt 1978). Define

$$
R_{i j}(\mathbf{r})=\int B_{i}(\mathbf{x}) B_{j}(\mathbf{x}+\mathbf{r}) \mathrm{d}^{3} x
$$

Then in Fourier space one finds

$$
\begin{aligned}
\widetilde{R}_{i j}(\mathbf{k}) & =\frac{1}{(2 \pi)^{3}} \int R_{i j}(\mathbf{r}) \mathrm{e}^{-\mathrm{i} \mathbf{k} \cdot \mathbf{r}} \mathrm{d}^{3} r \\
& =\widetilde{\mathbf{B}}_{\mathrm{k} i} \widetilde{\mathbf{B}}_{\mathrm{k} j}^{*} .
\end{aligned}
$$

We can then integrate over spherical shells in $k$ space as in equations 6 and 7 (with the help of 5):

$$
\begin{aligned}
M_{\mathrm{k}} & =\frac{1}{2} \int_{k-\text { shell }} \widetilde{R}_{i i} k^{2} \mathrm{~d} \Omega_{k}, \\
H_{\mathrm{k}} & =\int_{k-\text { shell }} \frac{-\mathrm{i} \epsilon_{\ell m n} k_{\ell} \widetilde{R}_{m n}}{k^{2}} k^{2} \mathrm{~d} \Omega_{k} .
\end{aligned}
$$

Thus the trace of $\widetilde{R}$ gives the energy spectrum, while the helicity spectrum comes from the anti-symmetric (and perpendicular to $\mathbf{k}$ ) part of $\widetilde{R}$. This relation between spectra and two point correlations will be discussed further in section 2.4.

\subsection{The relation between helicity spectrum and spatial structure}

The helicity spectrum measures the distribution of twist, shear, and linking on different length scales. It fails, however, in detecting spatial fluctuations of helicity. For example, consider the field

$$
\mathbf{B}=(\sin 2 x \cos 2 y,-\cos 2 x \sin 2 y, \sin x \sin y) .
$$

This field consists of twisted flux tubes aligned along the $z$ direction (see Figure 2). The helicity spectrum for this field is identically zero, i.e. $H_{\mathrm{k}}=0$ for all $\mathbf{k}$. While the field contains individual helicity containing tubes of alternating sign, the tubes have the same size. Thus the positive helicity exactly cancels the negative helicity at the same places in the spectrum. A quantity related to helicity which detects spatial 


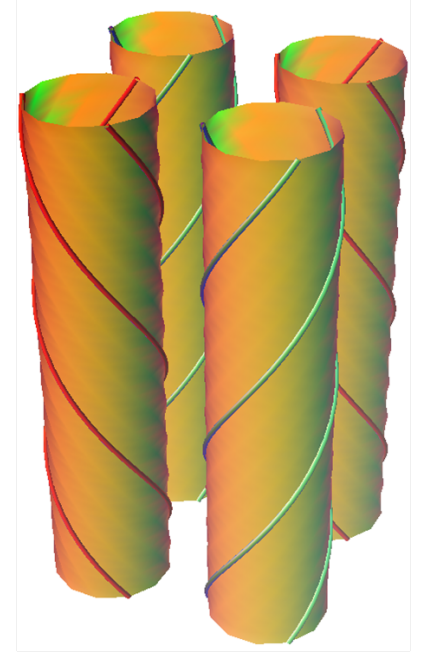

Figure 2.: A field whose helicity spectrum $H_{\mathrm{k}}=0$ for all $\mathbf{k}$.

distribution in weakly inhomogeneous turbulence has been proposed by Subramanian and Brandenburg (2006).

Helicity containing structures are inherently three dimensional. However, we can create a simple field which only varies in one dimension to investigate how a simple crossed field is represented in a Fourier spectrum:

$$
\mathbf{B}=\frac{1}{\sqrt{2 \pi} \sigma} \mathrm{e}^{-(z-w / 2)^{2} / 2 \sigma^{2}} \hat{\mathbf{x}}+\mathrm{e}^{-(z+w / 2)^{2} / 2 \sigma^{2}} \hat{\mathbf{y}}
$$

This field consists of two layers of flux separated by a distance $w$. Each layer has unit flux (per unit length in $x$ and $y$ directions) distributed with Gaussian profiles of width $\sigma$; their fields are perpendicular to each other.
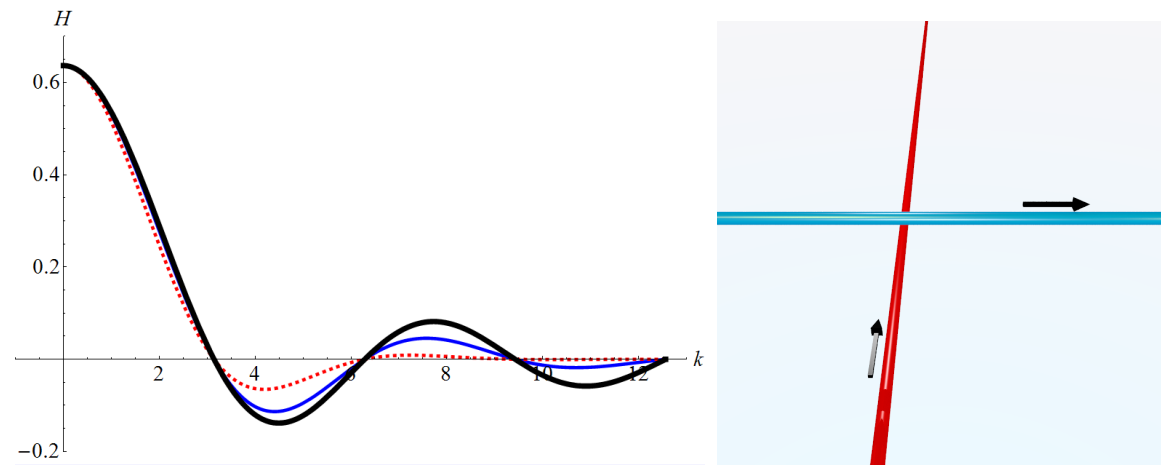

Figure 3.: Left: the helicity spectrum for two perpendicular thin slabs of magnetic flux. The separation between the slabs has been set at $w=1$. Curves are given for $\sigma=0.2$ (dashed), $\sigma=0.1$ (thin solid), and $\sigma \rightarrow 0$ (thick solid). Right: two crossed thin tubes of magnetic flux. The separation between the tubes is $w$. The helicity spectrum for this configuration is identical to that of the crossed slabs in limit of zero radius (thick solid curve).

Taking the Fourier transform in the $z$ direction of the above magnetic field, we have

$$
\widetilde{\mathbf{B}}_{\mathrm{k}}=\mathrm{e}^{\mathrm{i} k w / 2} \mathrm{e}^{-k^{2} \sigma^{2} / 2} \hat{\mathbf{x}}+\mathrm{e}^{-\mathrm{i} k w / 2} \mathrm{e}^{-k^{2} \sigma^{2} / 2} \hat{\mathbf{y}} .
$$


This gives a helicity spectrum

$$
H_{\mathrm{k}}=\frac{2}{\pi k} \mathrm{e}^{-k^{2} \sigma^{2}} \sin (k w)
$$

(see Figure 3). Here the envelope has width $\sigma / \sqrt{2}$ and the periodicity is $2 \pi / w$. The helicity spectrum broadens as the width $\sigma$ of the layers decreases; in the limit $\sigma \rightarrow 0$ it becomes

$$
\begin{aligned}
\mathbf{B} & =\delta(z-w / 2) \hat{\mathbf{x}}+\delta(z+w / 2) \hat{\mathbf{y}} \\
H_{\mathrm{k}} & =\frac{2}{\pi k} \sin (k w) .
\end{aligned}
$$

\subsection{Why does the helicity spectrum oscillate for localized flux?}

What is the origin of the $\sin (k w)$ factor in the helicity spectrum? We can better understand this effect by recalling the close relation between the helicity spectrum and the Fourier representation of the two-point correlation function $R_{i j}(\mathbf{r})$.

For the two slab field above (26), as variations only occur in $z$, we can write

$$
R_{12}(z)=\int B_{1}\left(z_{0}\right) B_{2}\left(z_{0}+z\right) \mathrm{d} z_{0}=\delta(z+w)
$$

Similarly $R_{21}(z)=\delta(z-w)$. Fourier transforms give

$$
\widetilde{R}_{12}=\frac{1}{2 \pi} \mathrm{e}^{-\mathrm{i} k w} ; \quad \widetilde{R}_{21}=\frac{1}{2 \pi} \mathrm{e}^{\mathrm{i} k w} .
$$

The one dimensional helicity spectrum analogous to (21) is

$$
\begin{aligned}
H_{\mathrm{k}} & =\frac{-\mathrm{i} \epsilon_{3 m n} k \widetilde{R}_{m n}(k)}{k^{2}}-\frac{-\mathrm{i} \epsilon_{3 m n} k \widetilde{R}_{m n}(-k)}{k^{2}} \\
& =\frac{1}{\mathrm{i} k}\left(\left(\widetilde{R}_{12}(k)-\widetilde{R}_{21}(k)\right)-\left(\widetilde{R}_{12}(-k)-\widetilde{R}_{21}(-k)\right)\right) \\
& =\frac{2}{\pi k} \frac{1}{2 \mathrm{i}}\left(\mathrm{e}^{\mathrm{i} k w}-\mathrm{e}^{-\mathrm{i} k w}\right)=\frac{2}{\pi k} \sin (k w),
\end{aligned}
$$

in accord with our previous calculation.

We can do a similar calculation for two crossed tubes (see right figure 3 ). Consider two thin unit flux tubes aligned along the $\mathrm{x}$ axis at $z=w / 2$, and along the $\mathrm{y}$ axis at $z=-w / 2$. Then one finds $R_{12}(z)=\delta(z+w)$ and $R_{21}(z)=\delta(z-w)$ as above (multiplied by flux squared). This leads to the same spectrum for $H_{\mathrm{k}}$.

Note that the above fields have net flux in the $\mathrm{x}$ and $\mathrm{y}$ directions. Despite the infinite domain, the helicity is finite. A more physically reasonable field with zero net flux has two oppositely directed tubes in the $x$ direction intertwined with two in the $y$ direction:

$$
\mathbf{B}=\delta(z-3 w / 2) \hat{\mathbf{x}}+\delta(z-w / 2) \hat{\mathbf{y}}-\delta(z+w / 2) \hat{\mathbf{x}}-\delta(z+3 w / 2) \hat{\mathbf{y}}
$$

This field still has an oscillatory spectrum, albeit with two modes of oscillation:

$$
H_{\mathrm{k}}=\frac{6}{\pi k} \sin (k w)-\frac{2}{\pi k} \sin (3 k w) .
$$




\section{Calculations}

\subsection{Method}

In this section, we consider the behavior of helicity in the stretch-twist-fold picture. Our aim is to understand how helicity is distributed over different scales, and how that distribution relates to the twist-writhe decomposition. We start with a magnetic flux tube of circular cross section and unit flux. The radius $a$ of the tube is held constant. At first, we consider a circular axis shape, then subject the shape to stretch, twist, and fold operations. The writhe is calculated employing the methods in (Berger and Prior 2006). Using this measurement of writhe, we determine the internal twist from equation (4).

In order to calculate the helicity and energy spectra, the tube is placed inside a three dimensional cubic grid (we employ $128^{3}$ ). The central axis of the tube is represented by $n$ points $(n=300$ seems sufficient). At each point on the grid the code first determines whether it is within a distance $a$ of the axis curve; if so then the magnetic field is determined. A fast Fourier transform then leads to a determination of the spectra.

One may worry that helicity is not always well-defined for periodic boxes (Berger 1997). This problem only presents itself when there is net flux in some direction; but our closed tubes do not have net flux in any direction.

Consider first a torus with minor radius $a$ and major radius $R$. The circular axis of the torus lies in a horizontal plane encircling the $z$ axis. In cylindrical coordinates a point is given by $(\rho, \phi, z)$. We will also need toroidal coordinates $(r, \theta, \phi)$ : here $r$ denotes the distance of a point from the circular axis of the torus, and $\theta$ denotes angle the short way around (with $\theta=0$ closest to the $z$ axis). We have

$$
\rho=R-r \cos \theta
$$

The field line equations are

$$
\frac{\mathrm{d} r}{B_{r}}=\frac{r \mathrm{~d} \theta}{B_{\theta}}=\frac{\rho \mathrm{d} \phi}{B_{\phi}} .
$$

So,

$$
B_{\theta}=\frac{r \mathrm{~d} \theta}{\rho \mathrm{d} \phi} B_{\phi}=\frac{r}{R-r \cos \theta} \frac{\mathrm{d} \theta}{\mathrm{d} \phi} B_{\phi} .
$$

If we replace $\phi$ by arc length $s$ along the toroidal axis, this becomes

$$
B_{\theta}=\frac{r R}{R-r \cos \theta} \frac{\mathrm{d} \theta}{\mathrm{d} s} B_{s}
$$

The field line derivative $\mathrm{d} \theta / \mathrm{d} s$ gives the rate of increase of twist $\mathcal{T}$ with arc length $s$, i.e.

$$
\frac{\mathrm{d} \theta}{\mathrm{d} s}=2 \pi \frac{\mathrm{d} \mathcal{T}}{\mathrm{d} s} \quad \text { (torus) }
$$

Next consider a thin flux tube of (almost) arbitrary shape. Locally, we can replace major radius $R$ in the above analysis by the curvature radius $R(s)$ of the central axis of the tube. We also lose the angle $\phi$, replacing it by arc length $s$ along the axis. The angle $\theta=0$ now points in the direction of the curvature vector. But in addition there will be an extra source of twist due to the torsion $\tau$ of the axis (Moffatt and Ricca 1992):

$$
\frac{\mathrm{d} \theta}{\mathrm{d} s}=2 \pi\left(\frac{\mathrm{d} \mathcal{T}}{\mathrm{d} s}-\tau\right)
$$


We assume twist (and thus parallel current) is uniformly distributed along the total arc length $L$ of the tube. In this case

$$
\frac{\mathrm{d} \mathcal{T}}{\mathrm{d} s}=\frac{H-\mathcal{W}}{L}
$$

Thus we have

$$
B_{\theta}=\frac{2 \pi r R(s)}{R(s)-r \cos (\theta)}\left(\frac{H-\mathcal{W}}{L}-\tau\right) B_{s}
$$

We keep $B_{s}$ uniform inside the tube. In order to find the upper limit for $a$, in the equation (42), the denominator $R-r \cos \theta$ should be more than zero. Hence, $R>r \cos \theta$ and for $\theta=0$, we should have $R>r$. Since we have $0 \leq r \leq a$, the radius of curvature $R(s)$ should always be larger than the minor radius $a$.

We employ a uniform radius $a=1.6$ in our study (the grid spacing is one). Larger radii make it more difficult to separate large and small scales in the spectra. Smaller radii are more subject to edge effects (i.e. the boundary of the flux tube is not properly resolved, resulting in significant changes to the spectra).

Helicity is measured in units of $\Phi^{2}$ (equivalently, we can set flux $\Phi=1$ and let $B_{s}=1 /\left(\pi a^{2}\right)$. Energy is measured in units of $\Phi^{2} / a$. In the graphs, wavenumber $k$ is measured in units of $k_{0}=2 \pi / N$, where $N$ is the size of the grid (we use $N=128$ ). Thus a wavelength of $\lambda$ corresponds to a wavenumber of $k=k_{0}^{-1}(2 \pi / \lambda)=N / \lambda$.

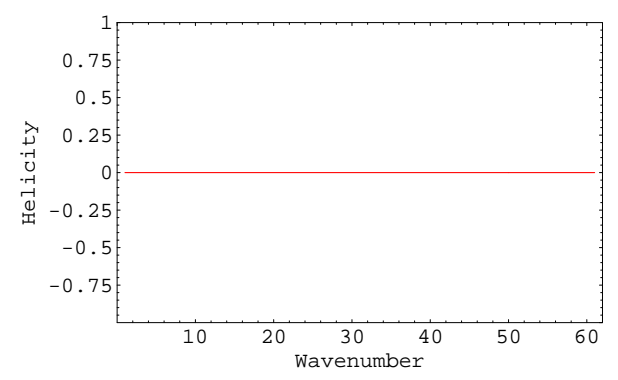

(a) $(H=0, \mathcal{W}=0, \mathcal{T}=0)$

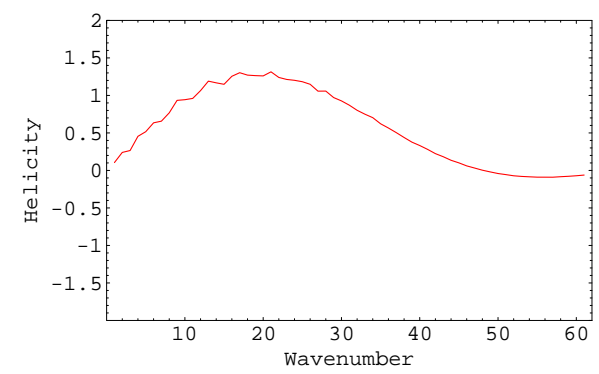

(c) $(H=0, \mathcal{W}=0, \mathcal{T}=1)$

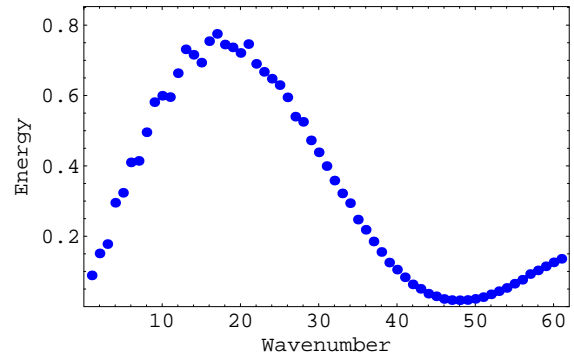

(b) $(H=0, \mathcal{W}=0, \mathcal{T}=0)$

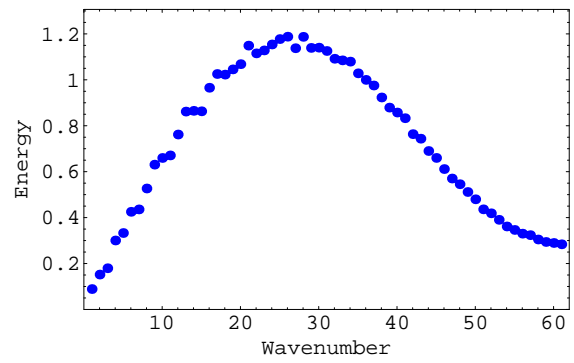

(d) $(H=0, \mathcal{W}=0, \mathcal{T}=1)$

Figure 4.: Spectra of a circular tube of radius $a=1.6$ placed in a $128^{3}$ grid. The tube has aspect ratio $=26$. (a) and (b): $H=\mathcal{W}=\mathcal{T}=0$. The magnetic helicity spectrum (a) shows the helicity remains zero for large and small scales (small and large wave numbers) despite any edge effects. The energy spectrum (b) shows a maximum at the wave number $k=17$ and a minimum at the wave number $k=48$. The edge effects prevent the energy in small scales from reaching zero value. (c) After increasing the internal twist of the circular tube to one, we see an initial increase in the helicity spectrum. (d) The corresponding increase in the energy spectrum of the circular tube. 
Table 1.: Mean wave numbers for the twisted tube of $5 \mathrm{a}$. The mean wave numbers are calculated in four different ways, employing weightings for energy, helicity, twist, and writhe.

\begin{tabular}{lcccc} 
& $\bar{k}(M)(\bar{\lambda}(M))$ & $\bar{k}(H)(\bar{\lambda}(H))$ & $\bar{k}\left(H_{+}\right)\left(\bar{\lambda}\left(H_{+}\right)\right)$ & $\bar{k}\left(H_{-}\right)\left(\bar{\lambda}\left(H_{-}\right)\right)$ \\
\hline $\mathcal{T}=-\mathcal{W}(\mathrm{H}=0)$ & $21.7(5.90)$ & $9.20(13.1)$ & $6.37(20.0)$ & $12.0(10.6)$ \\
$\mathrm{H}=\mathcal{W} \quad(\mathcal{T}=0)$ & $21.7(5.90)$ & $8.50(15.0)$ & $7.57(16.1)$ & $9.77(13.1)$ \\
$(\mathrm{H}=0)-(\mathcal{T}=0)(\mathcal{T}$ only $)$ & $21.7(5.89)$ & $22.0(5.81)$ & $56.4(2.27)$ & $21.5(5.95)$
\end{tabular}

\subsection{Circular tube}

Figure 4 shows the helicity and energy spectra for a circular magnetic flux tube with major radius 41.3 (aspect ratio 26). Note that edge effects give no fluctuations whatsoever to the helicity spectrum, although they do appear in energy (for wave numbers above 50).

The minor radius $a$ provides a significant length scale for the tube, which should affect the spectra. For the tube with internal twist, the azimuthal field reverse over one diameter, i.e. half a wavelength corresponds to $2 a$. Thus we infer a characteristic wavelength $\lambda_{a}=4 a$ and a corresponding wavenumber $k_{a}=N / 4 a$. For our parameters $k_{a}=20$, corresponding well to the peak of the helicity spectrum for the tube with internal twist (figure 4(c)). Also the energy spectrum for the $\mathcal{T}=0$ tube has a peak slightly below 20. This peak moves to the right for $\mathcal{T}=1$.

\subsection{Twisted tubes and folded tubes}

First we stretch the flux tube to twice its length preserving its volume. The helicity, writhe, and twist remain zero. Next we twist the flux tube, keeping the helicity zero. To easily see the effects on the spectra, we have twisted the tube axis by a considerable amount $3 \pi$ (Figure 5 ). The measurement of writhe shows $\mathcal{W}=1.152$. With the stretched and twisted tube, some new large-scale lengths become apparent. For example, the spacing between two points on opposite sides of the tube gives a scale $\lambda_{1} \approx 25.1$.

Here the helicity spectrum shows strong oscillations. The oscillation period is 5.36 , corresponding to a lengthscale of $128 / 5.36=23.9$. This is very close to the typical separation between neighboring sections of the tube $\lambda_{1} \approx 25$. Here we see an effect similar to that discussed in section 2.4: the two-point correlation function for the magnetic field of a thin tube will have sharp peaks at typical separation lengths. The helicity spectrum oscillates with a wavelength determined by these separation lengths.

We can separate the writhe and twist contributions to the helicity spectrum as follows: first, obtain the spectrum $H_{\mathrm{k}}(H=0)$ for a tube with zero net helicity. This includes equal and opposite twist and writhe contributions. Next obtain $H_{\mathrm{k}}(\mathcal{T}=0)$, the spectrum for a tube with the same shape but zero internal twist (thus zero internal electrical current). We will call this latter spectrum the writhe helicity spectrum. Finally, the twist helicity spectrum will be defined as the difference between the two, $H_{\mathrm{k}}(H=0)-H_{\mathrm{k}}(\mathcal{T}=0)$. The final two figures in 5 show these two spectrums. Figure 5 d shows that writhe helicity takes both positive and negative values at large scales and at small scales it reaches zero. Thus while it is concentrated at large scales, as expected, it also displays large oscillations.

Figure 6 shows the spectra of the folded tube (bottom middle tube in figure 1 ) with writhe $\mathcal{W}=1.118$. Qualitatively the spectra are similar to the twisted tube spectra. Here the oscillations in the helicity spectrum have a smaller frequency of about 3.6, corresponding to a lengthscale of 36 . Taken as a whole, the folded tube has a size of about $36 \times 55 \times 33$.

Table 1 provides the mean wave numbers for the twisted tube. Using these values, we find the wave length $\bar{\lambda}_{k}=128 / \bar{k}$, where $\bar{k}$ is the mean wavenumber given some weighting. Thus if we weight by energy,

$$
\bar{k}(M)=\frac{\sum k M_{\mathrm{k}}}{\sum M_{\mathrm{k}}}
$$




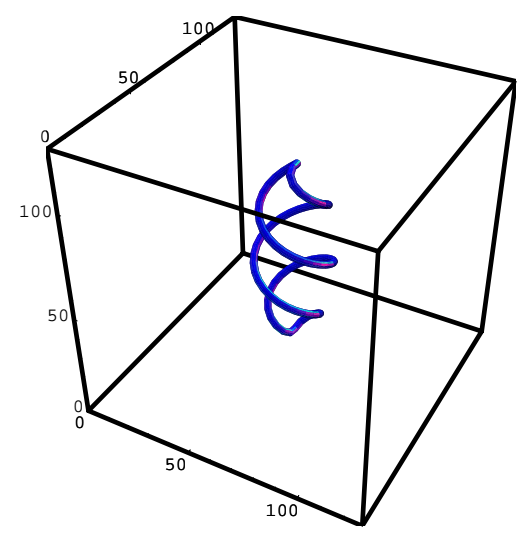

(a)

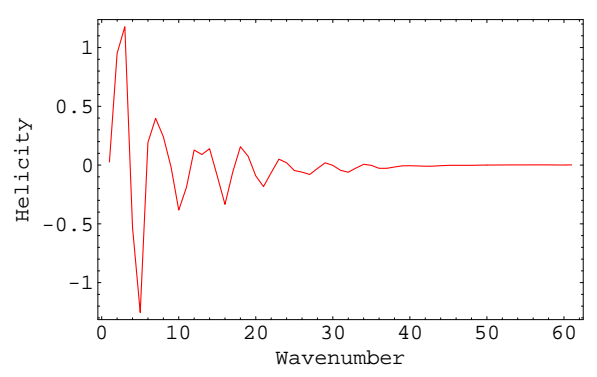

(b) $(H=0, \mathcal{W}=1.152, \mathcal{T}=-1.152)$

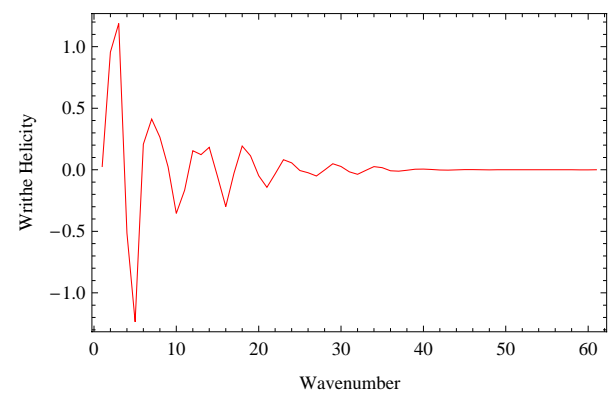

(d) Writhe helicity spectrum

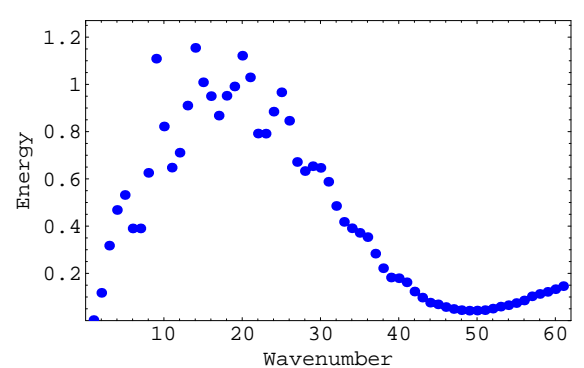

(c) $(H=0, \mathcal{W}=1.152, \mathcal{T}=-1.152)$

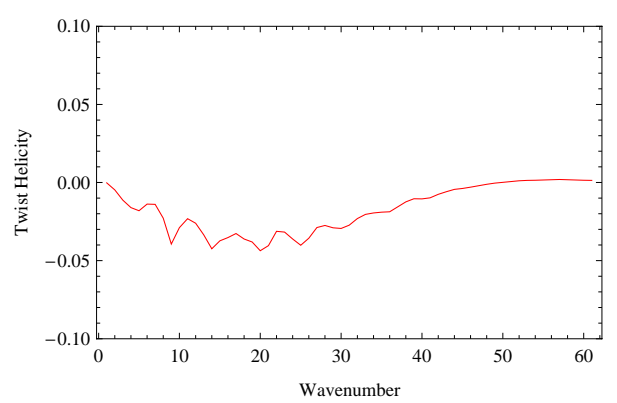

(e) Twist helicity spectrum

Figure 5.: Spectra of a tube turned through an angle of $3 \pi(\mathcal{W}=1.152)$. Figure (b) gives the helicity spectrum for the tube with total helicity $H=0$. Between the wave number $k=1$ to $k=4$ (indicator of large scale) the helicity is positive. Then there is a sharp decrease in the value of helicity, having negative value and from then on the helicity oscillates between negative and positive values. From Fourier analysis the oscillation frequency is 5.36. Figure (c) shows the energy distribution of the twisted flux tube. (d) The writhe helicity spectrum. (e) The twist helicity spectrum. Note that the range for graph (e) is much smaller than graph (d).

while if we weight by helicity (actually $\left|H_{\mathrm{k}}\right|$ )

$$
\bar{k}(H)=\frac{\sum k\left|H_{\mathrm{k}}\right|}{\sum\left|H_{\mathrm{k}}\right|} .
$$

We then compare the values of $\bar{\lambda}_{k}$ with $\lambda_{a}=4 a=4 \times 1.6=6.4$. We look at three cases: zero helicity, writhe helicity, and twist helicity. When using energy as the weighting factor for all three, $\bar{\lambda}_{k}(M)$ is very close to $\lambda_{a}$. In the second column, with helicity $\left|H_{\mathrm{k}}\right|$ as the weighting factor, there is a clear separation between the twist helicity (at a length scale near $\lambda_{a}$ ) and the writhe helicity (at two and half times larger 


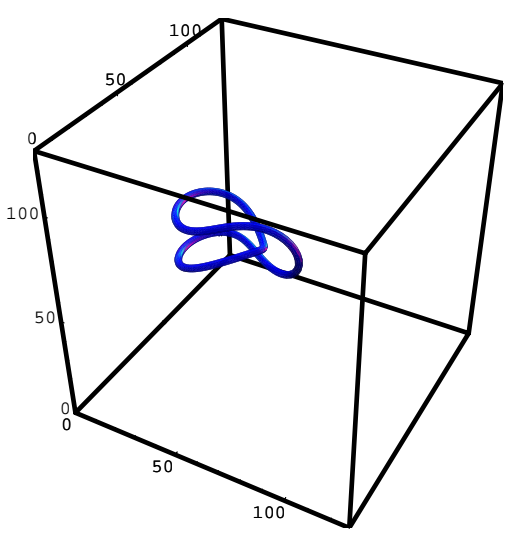

(a)

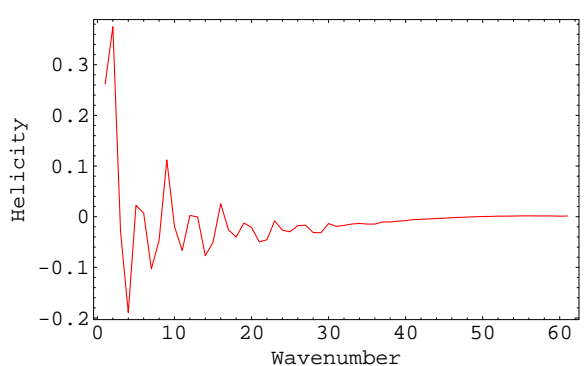

(b) $(H=0, \mathcal{W}=1.118, \mathcal{T}=-1.118)$

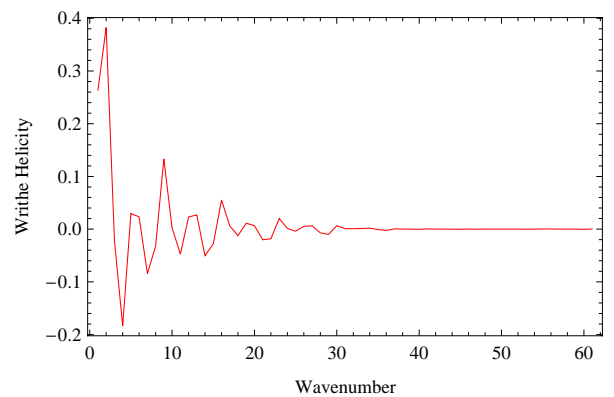

(d) Writhe helicity spectrum

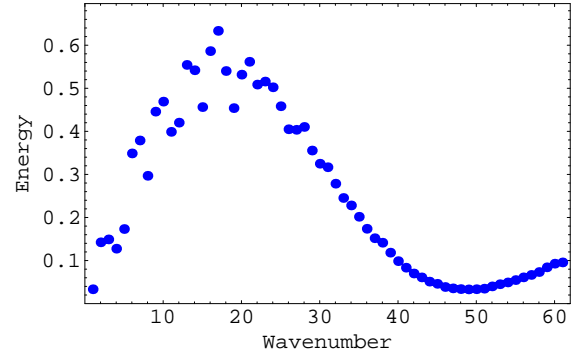

(c) $(H=0, \mathcal{W}=1.118, \mathcal{T}=-1.118)$

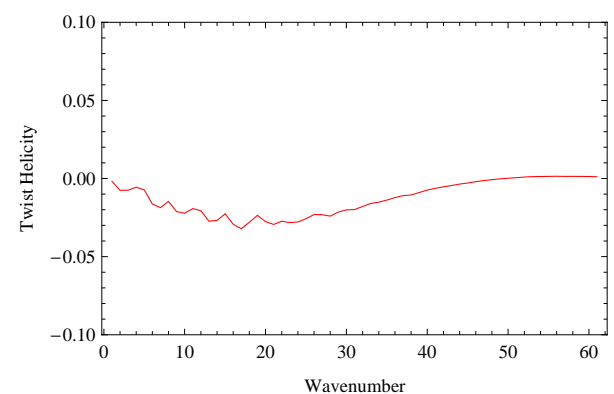

(e) Twist helicity spectrum

Figure 6.: Spectra for a folded tube. Figure (b) gives the helicity spectrum. For wave numbers $k=1$ to $k=4$ the helicity is positive. Then there is a sharp decrease in the value of helicity and from then on helicity oscillates between negative and positive values, with a frequency of 3.58. (c) Shows the energy distribution of the folded flux tube. (d) The writhe helicity spectrum. (e) The twist helicity spectrum. Note that the range for graph (e) is much smaller than graph (d).

scale). We can try to sharpen this separation by weighting with only the positive values of the helicity spectrum $\left(H_{+}\right)$or negative values $\left(H_{-}\right)$. Here the positive writhe goes to slightly larger scales (slightly smaller $\bar{k}$ ). The negative writhe comes slightly closer to $\lambda_{a}$. The positive part of the twist helicity is at very large wave numbers, suggesting perhaps some slight edge effects or numerical noise.

\subsection{The writhe spectrum of compact curves}

Maggioni and Ricca (2006) present several families of analytic closed curves, and give a detailed analysis of the behaviour of both intrinsic quantities such as curvature and torsion, and global quantities such as writhe and elastic energy. There is a possible application in biology: many long biological molecules must be strongly coiled in order to fit inside cells. This can require multiple levels of supercoiling. We note that 

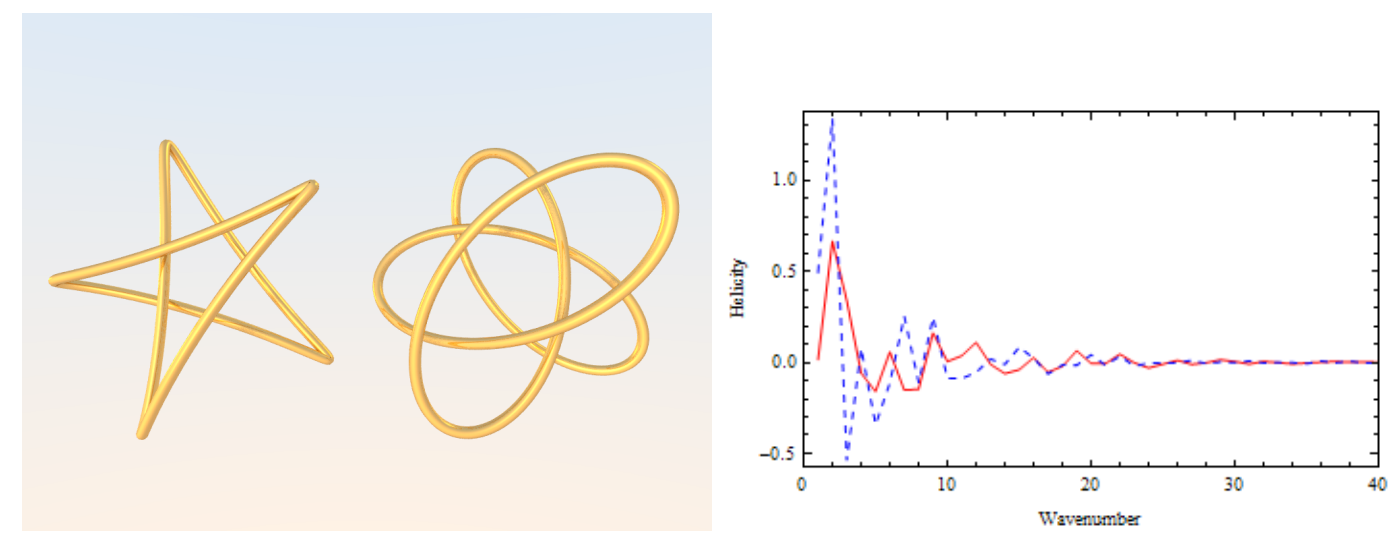

Figure 7.: Two hypocycloids with their writhe spectra. On the left $\beta=0.6$ with $\mathcal{W}=1.1$ (red solid line), while on the right $\beta=2$ with $\mathcal{W}=2.0$ (blue dashed line).

the technique of finding a Fourier spectrum for the writhe as presented here may complement this study. A Fourier analysis shows how the writhe distributes itself on different length scales. As a simple example, figure 7 shows two realizations of the hypocycloid curve described by

$$
\mathbf{x}(t)=(\cos 2 t+\beta \cos 3 t, \sin 2 t-\beta \sin 3 t, \beta \sin t),
$$

with free parameter $\beta$. The usual oscillations still occur with these shapes; it may be that for more irregular shapes the oscillations would smooth out.

\section{Conclusions}

This paper has analyzed the influence of twist and writhe numbers on helicity and energy spectra in the stretch-twist-fold dynamo. Calculating the writhe number of a flux tube has two purposes. First, it assists us in finding out how much the internal twist changes as the tube is distorted. This greatly simplifies the modeling of a thin flux tube. Second, the writhe provides a valuable diagnostic for large scale structure. We have compared this diagnostic with the more usual one: examining power spectra at low wave numbers. We showed that there is a strong correlation between the helicity distribution over different scales and the twist-writhe decomposition. This correlation shows up in the mean wave number calculations (table 1). As expected (Brandenburg and Subramanian 2005, Gilbert 2002), twist helicity appears at relatively large wave numbers, while writhe helicity appears at smaller wave numbers. The mean wavenumber for twist helicity corresponds roughly to a wavelength of four times the radius of the tube. However, the helicity spectrum for writhe helicity is more complicated. While there is net power at low wave numbers, the spectrum also oscillates with a wide envelope. Although there is a great deal of cancellation between positive and negative helicity, there is no clear boundary between negative helicity at large scales and positive helicity at small scales.

Of course, an ensemble of flux tubes of many sizes will all have their own oscillating spectra, somewhat out of phase with each other. Thus, in such an ensemble the oscillations might cancel, leaving only the envelope. Similarly, irregular curves may have oscillations with a broad range of frequencies, again with the result of smoothing out the writhe spectrum. However the large gap between mean wave numbers for twist and writhe helicity will likely survive.

\section{Acknowledgments}

We are very grateful to Andrew Gilbert, Pascal Démoulin and Chris Prior for helpful discussions. 


\section{REFERENCES}

Archontis, V., Moreno-Insertis, F., Galsgaard, K., Hood, A. and O'Shea, E. (2004). Emergence of magnetic flux from the convection zone into the corona, Astronomy and Astrophysics 426: 1047-1063.

Bao, S. and Zhang, H. (1998). Patterns of current helicity for the twenty-second solar cycle, The Astrophysical Journal 496: L43-L46.

Basu, S. (1997). Seismology of the base of the solar convection zone, Monthly Notices of the Royal Astronomical Society 288: 572-584.

Beck, R., Brandenburg, A., Moss, D., Shukurov, A. and Sokoloff, D. (1996). Galactic magnetism: Recent developments and perspectives, Annu. Rev. Astron. Astrophys 34: 155-206.

Berger, M. A. (1997). Magnetic helicity in a periodic domain, Journal of Geophysical Research 102: 26372644.

Berger, M. A. and Field, G. B. (1984). The topological properties of magnetic helicity, Journal of Fluid Mechanics 147: 133-148.

Berger, M. A. and Prior, C. (2006). The writhe of open and closed curve, J. Phys. A: Math. Gen. 39: 8321-8348.

Berger, M. A. and Ruzmaikin, A. (2000). Rate of helicity production by solar rotation, Journal of Geophysical Research 105: 10481-10490.

Blackman, E. G. and Brandenburg, A. (2003). Doubly helical coronal ejections from dynamos and their role in sustaining the solar cycle, Astrophys. J. Lett. 584: L99-L102.

Blackman, E. G. and Field, G. B. (2000). Coronal activity from dynamos in astrophysical rotators, Monthly Notices of the Royal Astronomical Society 318: 724-732.

Brandenburg, A. (2001). The inverse cascade and nonlinear alpha effect in simulations of isotropic helical hydromagnetic turbulence, The Astrophysical Journal 550: 824-840.

Brandenburg, A., Dobler, W. and Subramanian, K. (2002). Magnetic helicity in stellar dynamos: new numerical experiments, Astronomische Nachrichten 323: 99-122.

Brandenburg, A. and Subramanian, K. (2005). Astrophysical magnetic fields and nonlinear dynamo theory, Physics Reports 417: 1-209.

Brown, M. R., Canfield, R. C. and Pevtsov, A. A. (1999). Magnetic helicity in space and laboratory plasmas, Geophys. Monograph 111, American Geophysical Union, Florida .

Childress, S. and Gilbert, A. D. (1995). Stretch, twist, fold: the fast dynamo, Berlin: Springer .

Chou, D. Y. and Fisher, G. H. (1989). Dynamics of anchored flux tubes in the convection zone. i - details of the model, The Astrophysical Journal 341: 533-548.

Choudhuri, A. R. and Gilman, P. A. (1987). The influence of the coriolis force on flux tubes rising through the solar convection zone, The Astrophysical Journal 316: 788-800.

Călugăreanu (1961). On isotopy classes of three dimensional knots and their invariants, Czechoslovak Math. J. T11 588.

Démoulin, P., Mandrini, C. H., van Driel-Gesztelyi, L., Lopez Fuentes, M. C. and Aulanier, G. (2002). The magnetic helicity injected by shearing motions, Solar Physics 207: 87-110.

DeVore, C. R. (2000). Magnetic helicity generation by solar differential rotation, The Astrophysical Journal 539: $944-953$.

D'Silva, S. and Choudhuri, A. R. (1993). A theoretical model for tilts of bipolar magnetic regions, Astronomy and Astrophysics 272: 621-633.

Epple, M. (1998). Orbits of asteroids, a braid, and the first link invariant, Math. Intelligencer 20: 45-52.

Fan, Y., Fisher, G. H. and Deluca, E. E. (1993). The origin of morphological asymmetries in bipolar active regions, The Astrophysical Journal 405: 390-401.

Field, G. B. and Blackman, E. G. (2002). Dynamical quenching of the $\alpha^{2}$ dynamo, The Astrophysical Journal 572: 685-692.

Gilbert, A. (2002). Magnetic helicity in fast dynamos, Geophysical and Astrophysical Fluid Dynamics 96(2): 135-151.

Hale, G. E., Ellerman, F., Nicholson, S. B. and Joy, A. H. (1919). The magnetic polarity of sun-spots, The Astrophysical Journal 49: 153-178. 
Howe, R., Christensen-Dalsgaard, J., Hill, F., Komm, R. W., Larsen, R. M., Schou, J., Thompson, M. J. and Toomre, J. (2000). Deeply penetrating banded zonal flows in the solar convection zone, The Astrophysical Journal 533: L163-L166.

Krause, F. (1993). The cosmic dynamo: From $t=-\infty$ to cowling's theorem. a review on history, The cosmic dynamo: proceedings of the 157th Symposium of the International Astronomical Union held in Potsdam; F.R.G.; September 7-11; 1992. Edited by F. Krause, K. H. Radler, and Gunther Rudiger. International Astronomical Union. Symposium no. 157; Kluwer Academic Publishers; Dordrecht pp. 487-499.

Kronberg, P. P. (1994). Extragalactic magnetic fields, Rep. Prog. Phys 57: 325-382.

Kulsrud, R. M. (1999). A critical review of galactic dynamos, Annual Review of Astronomy and Astrophysics 37: 37-64.

Leka, K. D., Canfield, R. C., McClymont, A. N. and Van Driel Gesztelyi, L. (1996). Evidence for currentcarrying emerging flux, The Astrophysical Journal 462: 547-560.

Longcope, D. W., Fisher, G. H. and Arendt, S. (1996). The evolution and fragmentation of rising magnetic flux tubes, The Astrophysical Journal 464: 999-1011.

Longcope, D. W. and Klapper, I. (1997). Dynamics of a thin twisted flux tube, The Astrophysical Journal 488: $443-453$.

Longcope, D. W., Linton, M. G., Pevtsov, A. A., Fisher, G. H. and Klapper, I. (1999). Twisted flux tubes and how they get that way, In magnetic helicity in space and laboratory plasmas, ed. M. R. Brown, R. C. Canfield, A. A. Pevtsov, Geophysical monograph series, AGU Washington, D.C 111: 93.

Longcope, D. W., Ravindra, B. and Barnes, G. (2007). Determining the source of coronal helicity through measurements of braiding and spin helicity fluxes in active regions, The Astrophysical Journal 668: $571-585$.

Maggioni, F. and Ricca, R. L. (2006). Writhing and coiling of closed filaments, Proceedings-Royal Society. Mathematical, Physical and Engineering Sciences 462: 3151-3166.

Moffatt, H. K. (1969). The degree of knottedness of tangled vortex lines, Journal of Fluid Mechanics 35: $117-129$.

Moffatt, H. K. (1978). Magnetic field generation in electrically conducting fluids, Cambridge University Press .

Moffatt, H. K. and Proctor, M. R. E. (1985). Topological constraints associated with fast dynamo action, Journal of Fluid Mechanics 154: 493-507.

Moffatt, H. K. and Ricca, R. L. (1992). Helicity and the călugăreanu invariant, Proceedings-Royal Society. Mathematical and Physical Sciences 439: 411-429.

Moreno-Insertis, F. and Emonet, T. (1996). The rise of twisted magnetic tubes in a stratified medium, The Astrophysical Journal 472: L53-L56.

Ossendrijver, M. (2003). The solar dynamo, The Astronomy and Astrophysics Review 11: 287-367.

Parker, E. N. (1979). Cosmical magnetic fields, Clarendon Press .

Pevtsov, A. A., Canfield, R. C. and Metcalf, T. R. (1995). Latitudinal variation of helicity of photospheric fields, The Astrophysical Journal 440: L109-L112.

Pevtsov, A. A. and Latushko, S. M. (2000). Current helicity of the large scale photospheric magnetic field, The Astrophysical Journal 528: 999-1003.

Pohl, W. F. (1980). DNA and differential geometry, Math. Intelligencer 3: 20-27.

Rust, D. M. and Kumar, A. (1996). Evidence for helically kinked magnetic flux ropes in solar eruptions, The Astrophysical Journal 464: L199-L202.

Rust, D. M. and LaBonte, B. J. (2005). Observational evidence of the kink instability in solar filament eruptions and sigmoids, The Astrophysical Journal 622: L69-L72.

Schou, J., Christensen-Dalsgaard, J. and Thompson, M. J. (1992). The resolving power of current helioseismic inversions for the sun's internal rotation, The Astrophysical Journal 385: L59-L62.

Schussler, M. (1979). Magnetic buoyancy revisited: Analytical and numerical results for rising flux tubes, Astronomy and Astrophysics 71: 79-91.

Spruit, H. C. (1981). Motion of magnetic flux tubes in the solar convection zone and chromosphere, Astronomy and Astrophysics 98: 155-160. 
Thompson, M. J., Christensen-Dalsgaard, J., Miesch, M. S. and Toomre, J. (2003). The internal rotation of the sun, Annual Review of Astronomy and Astrophysics 41: 599-643.

Tobias, S. M. (2002). The solar dynamo, Phil. Trans. R. Soc. Lond. A 360: 2741-2756.

Torok, T. and Kleim, B. (2003). The evolution of twisting coronal magnetic flux tubes, Astronomy and Astrophysics 406: 1043-1059.

Torok, T. and Kleim, B. (2005). Confined and ejective eruptions of kink-unstable flux ropes, The Astrophysical Journal 630: L97-L100.

Torok, T., Kleim, B. and Titov, V. S. (2004). Ideal kink instability of a magnetic loop equilibrium, Astronomy and Astrophysics 413: L27-L30.

Tsinganos, K. (1980). Sunspots and the physics of magnetic flux tubes. x - on the hydrodynamic instability of buoyant fields, The Astrophysical Journal 239: 746-760.

Vainshtein, S. I., Sagdeev, R. Z. and Rosner, R. (1997). Stretch-twist-fold and ABC nonlinear dynamos: Restricted chaos, Physical Review E 56: 1605-1622.

Vainshtein, S. I., Sagdeev, R. Z., Rosner, R. and Kim, E. J. (1996). Fractal properties of the stretch-twistfold magnetic dynamo, Physical Review E 53: 4729-4744.

Vainshtein, S. I. and Zeldovich, Y. B. (1972). Origin of magnetic fields in astrophysics, Soviet Physics Uspekhi 15: 159-172.

Valori, G., Kliem, B. and Keppens, R. (2005). Exploration of a nonlinear force-free field containing a highly twisted magnetic loop, Astronomy and Astrophysics 433: 335-347.

van Ballegooijen, A. A. (1999). Photospheric motions as a source of twist in coronal magnetic fields, Magnetic helicity in space and laboratory plasmas, Geophysical Monograph Series, ed. by M. R. Brown, R. C. Canfield, A. A. Pevtsov 111: 213-220.

White, J. H. (1969). Self linking and the gauss integral in higher dimensions, American Journal of Mathematics 91: 693-728.

Widrow, L. M. (2003). Origin of galactic and extragalactic magnetic fields, Rev. Mod. Phys 74: 775-823.

Yoshizawa, A., Itoh, S.-I., Itoh, K. and Yokoi, N. (2004). Dynamos and MHD theory of turbulence suppression, Plasma Phys. Control. Fusion 46: R25-R94.

Yousef, T. A. and Brandenburg, A. (2003). Relaxation of writhe and twist of bi-helical magnetic field, Astronomy and Astrophysics 407: 7-12.

Zeldovich, Y. B., Ruzmaikin, A. A. and Sokoloff, D. D. (1983). Magnetic fields in astrophysics, Gordon and Breach, New York .

Zweibel, E. G. and Heiles, C. (1997). Magnetic fields in galaxies and beyond, Nature 385: 131-137. 